\title{
La colaboración como pilar esencial de la Inclusión Educativa en el aula de clase
}

\author{
Collaboration as an essential pillar of educational \\ inclusion in the classroom
}

\section{A colaboração como pilar essencial da Inclusão Educativa na sala de aula}

\author{
Johanna Fernanda Mendoza Chala \\ Licenciada en Pedagogía Infantil \\ Universidad Surcolombiana \\ johannamendoza2003@hotmail.com
}

\section{Resumen}

La educación a nivel general no ha escapado a los diferentes cambios que se han instaurado en el transcurso de la historia del ser humano. Es más que evidente el vivenciar, desde el aula particularmente, la manera cómo se enseña y cómo se aprende en relación con siglos pasados. Como parte importante de este proceso, se debe pensar en la metodología, misma que debe considerar las actividades interactivas entre los estudiantes y entre estos y los docentes, en donde prime la cooperación como valor esencial en el aula y que favorezca la inclusión de todas las personas considerando su diversidad. Es así, entonces, que surge la necesidad de reflexionar acerca de una práctica colaborativa que favorezca la inclusión en el aula de clase. Para lo cual, se plantean algunos aspectos necesarios como los principios de la inclusión, las condiciones del aula inclusiva y las características de los diversos estamentos involucrados, esencialmente, los docentes quienes son los llamados a ejercer una práctica inclusiva real, a partir de la cooperación como valor esencial de la propuesta.

Palabras claves: Colaboración, inclusion educative, principios de la inclusion, educar, enseñar.

\begin{abstract}
Education at a general level has not escaped the different changes that have been introduced in the course of human history. It is more than evident to experience, from the classroom in particular, the way in which it is taught and how it is learned in relation to past centuries. As an important part of
\end{abstract}


this process, the methodology must be considered, which should consider the interactive activities between students and between them and teachers, where cooperation is an essential value in the classroom and that favors the inclusion of all people considering their diversity. It is thus, then, that the need arises to reflect on a collaborative practice that favors inclusion in the classroom. For which, some necessary aspects are raised such as the principles of inclusion, the conditions of the inclusive classroom and the characteristics of the various levels involved, essentially, the teachers who are called to exercise a real inclusive practice, based on cooperation as an essential value of the proposal.

Keywords: Collaboration, educational inclusion, principles of inclusion, educate, teach.

\section{Resumo}

A educação a nível geral não escapou às diferentes mudanças que se instauraram no curso da história do ser humano. É mais que evidente o vivenciar, desde a sala de aula particularmente, a maneira como se ensina e como se aprende em relação com séculos passados. Como parte importante deste processo, deve-se pensar na metodologia, mesma que deve considerar as atividades interativas entre os estudantes e entre estes e os docentes, onde privilegie a cooperação como valor essencial na sala de aula e que favoreça a inclusão de todas as pessoas considerando sua diversidade. É assim, então, que surge a necessidade de refletir sobre uma prática colaborativa que favoreça a inclusão na sala de aula. Para isso, colocam-se alguns aspectos necessários como os princípios da inclusão, as condições da sala inclusiva e as características dos diversos estamentos envolvidos, essencialmente, os docentes que são chamados a exercer uma prática inclusiva real, com base na cooperação como valor essencial da proposta.

Palavras-chave: colaboração, inclusão educativa, princípios da inclusão, educar, ensinar.

\section{Introducción}

La educación requiere una serie de cambios y modificaciones para que se vuelva más inclusiva (Crisol Moya, Martínez, \& El Homrani, 2015) y así, pueda cumplir con los derechos de la población en condición social de discapacidad como cualquier otro estudiante (Montáncez, Jornet, Perales, Carrillo, \& Wilches, 2017). Así, aparece la Educación especial como un proceso que busca:

Un servicio de apoyo a la educación general que estudia de manera global e integrada los procesos de enseñanza-aprendizaje, y que se define por los apoyos necesarios y nunca por las limitaciones del alumnado con el fin de lograr el máximo desarrollo personal y social de las personas en edad escolar que presenta Necesidades Educativas Especiales, independientemente de la razón (p. 255).
La educación inclusiva incluye la idea esencial de considerarla como un proceso que aborda la diversidad de necesidades a través de la participación activa de todos los involucrados y no solo de los estudiantes, en un proceso eminentemente colaborativo. Esto requiere una serie de adaptaciones a todo nivel: personal, institucional, social.

Otros autores (Matsura, 2008, citando (Crisol Moya et al., 2015) señalan que la educación inclusiva es un enfoque para mejorar la calidad de la educación, para responder a la diversidad y promover el aprendizaje, apoyando y estimulando a todos los involucrados a participar en el proceso.

Sin embargo, plantea la imperiosa necesidad de llevar a cabo ciertas modificaciones que deben pensarse en función de aspectos tales como la cooperación, la solidaridad, el interés por la 
comunidad, el respeto y valoración de las diferencias y que podrían incluir también la investigación reflexiva. Todos aspectos que invitan a realizar modificaciones creativas en la institución con la finalidad de entender y asumir la diversidad como parte de la misión de la entidad, considerando la colaboración como un eje de esta propuesta.

Se trata de dar valor a la colaboración en la diversidad, como un aspecto importante de la organización; así, las actividades propuestas a todo nivel, uno de los cuales corresponde al currículo responden a una diversidad de personas en un proceso colaborativo y participativo de construcción personal y social.

Un aspecto muy importante de considerar la colaboración como parte esencial de la inclusión es que la diversidad parte de la interculturalidad (Terrón-Caro, Cárdenas-Rodríguez, \& Rodríguez-Casado, 2017) con la finalidad de que las personas adquieran una clara conciencia sobre la pluralidad en la que están inmersos.

Partiendo de estas ideas iniciales, entonces, se postula la importancia de la colaboración para desarrollar y promover la inclusión en el aula. Para concretar este tema, se abordarán tres aspectos importantes al momento de pensar la colaboración para lograr la inclusión. Estos aspectos son los principios del aula inclusiva, las condiciones del aula inclusiva, en la que se considerará el clima escolar como aspecto esencial y los actores de la inclusión considerando a los docentes, la familia y la comunidad como partícipes importantes del proceso colaborativo que desarrolla la inclusión. A continuación, su desarrollo.

\section{Principios del aula inclusiva}

Un aula inclusiva para considerarse como tal, debe fundamentarse en ciertos principios (Crisol Moya et al., 2015) referentes a aspectos sociales e institucionales que determinan cambios y transformaciones necesarias en los que todos deben colaborar para poder concretarlos. Es así como algunos autores plantean los principios para concretizar un aula inclusiva (Crisol Moya et al., 2015), los cuales van a generar mayor bienestar en las personas que se beneficien de su consideración y aplicación en entornos educativos (Lázaro-Visa, Palomera, Briones, Fernández-Fuentes, \& Fernández-Rouco, 2019). Estos principios se los puede asociar con varios niveles como son el social, el institucional y el personal (Briones, 2015).

A nivel social es muy importante que existan principios que determinen la generación de una política educativa, el financiamiento adecuado de las actividades, la evaluación del sector en el que se ubica el centro, especialmente, en lo que se refiere a los accesos posibles y las facilidades o dificultades que presenta y, el establecimiento de colaboración entre centros tomando en consideración el apoyo del gobierno y de los organismos centrales.

En cuanto se refiere al nivel institucional, los principios formulados, deben proponer la realización de cambios para favorecer la inclusión, tales como la importancia de identificar necesidades, planificar el cambio, ejecutarlo, evaluarlo y realizar las adaptaciones más adecuadas. Estos aspectos se han venido potenciando en Colombia, puesto que existen ya propuestas (Guerrero, Crissien, \& Aniagua, 2017) que pretenden la creación de un currículo inclusivo gracias al análisis del Proyecto Educativo Institucional (PEI) de una institución educativa, basándose en los lineamientos de la Guía 34 expedida por el Ministerio de Educación Nacional de Colombia.

Un cambio muy importante a llevar a cabo, es la modificación de la misión definida por la institución, en la que se explicite claramente el tema de la inclusión y que se lleve a cabo gracias a un liderazgo y actitud de todo el personal (Muñoz, Lucero, Cornejo, Muñoz, \& Araya, 2014). Estos aspectos contribuyen a una convivencia más pacífica, tolerante y colaborativo en la comunidad educativa, caracterizada por el respeto a las normas, la confianza de los 
estudiantes con los docentes y las actividades compartidas con las familias.

Estos principios deben efectivizarse en el fomento de ciertas condiciones en el aula de clase, que favorezcan la inclusión, por lo que se consideran a continuación.

\section{Condiciones del aula inclusiva}

Además de las condiciones sociales y de las instituciones educativas que deben modificarse para alcanzar una verdadera inclusión en el aula, según González Ledesma, (2012) es importante considerar el microsistema del aula misma y el clima que se construye en ella y que puede o no, favorecer la inclusión.

Es posible identificar algunas características (Crisol Moya et al., 2015), tales como: la concepción y desarrollo del currículo común centrado en la diversidad, la valoración positiva de la diversidad, la organización social del aula: respeto a las diferencias, el aprendizaje cooperativo y flexible, los diferentes enfoques para la evaluación y la participación activa del alumnado, que se constituyen en elementos indispensables en la construcción de la inclusión en el aula y en la institución.

Además, hay que considerar otros aspectos importantes como: las actitudes de los docentes, la formación para facilitar la inclusión (Rodríguez, 2018), el acceso a recursos adecuados, apoyo interno en la escuela y externo de los padres de familia.

Tomando en consideración estos elementos, entonces, es posible pensar en algunas características que marcan y definen a un aula inclusiva (Montáncez et al., 2017):

La filosofía asumida por el docente acerca del aula y sus posibilidades. Si el docente desea proponer un aula inclusiva debe asumir esta filosofía, que muestra apertura hacia las diferencias y en la que se valora la diversidad (D. Ortiz Granja, 2015) (D. N. Ortiz Granja \&
Joaqui Robles, 2017).

La formulación de reglas en el aula que facilita las actividades y en las cuales se comunica abierta e intencionalmente, los derechos y deberes de cada miembro existente en el aula.

La investigación acerca de las características de los estudiantes (Balongo \& Mérdida, 2015) con la finalidad de que pueda proporcionar el apoyo y la ayuda necesaria a aquellos que tienen características particulares.

También es necesaria una comunicación efectiva, caracterizada por un abordaje sincero y transparente de temas álgidos para las personas, como los prejuicios y los estereotipos que solo conducen a la exclusión de ciertas personas. Es importante enfatizar en los aspectos comunes más que en las diferencias; sin embargo, se deben tomar en cuenta estas para llevar a cabo la labor docente.

Además, también es necesario considerar que las actividades a realizarse en el aula, tengan distinto nivel de dificultad (Giangreco, et al., 1994), para acercar a todos al mismo problema de forma paulatina. La conformación de grupos heterogéneos de estudiantes contribuye a lograr este objetivo puesto que cada uno aporta sus conocimientos y destrezas para resolver los problemas planteados. La finalidad implícita de todo esto, es lograr una experiencia educativa equilibrada y colaborativa en todos los aspectos: personales, relacionales, organizacionales e incluso sociales.

Cuando la institución considera estos diversos elementos implicados en un quehacer inclusivo colaborativo, promueve la construcción de un clima institucional adecuado para el desempeño de todos los actores del sistema.

Es así, que se puede definir "clima escolar" (Pineda, Granizo, \& Zúniga, 2019) como "el ambiente dentro del cual se desarrolla el proceso educativo, los elementos del clima escolar 
depende en gran parte del factor humano, las situaciones y los hechos son consecuencias de acciones realizadas por las personas" (p. 27). Existen muchos elementos que conforman el clima de un aula, sin embargo, hay algunos aspectos que son claves como las actitudes de cada integrante del sistema educativo, así como el ambiente que les rodea (Pineda et al., 2019).

Se trata de una variable clave para potenciar los recursos de los estudiantes y que tengan un buen rendimiento. Además, modela el comportamiento de las personas, desarrollando un cúmulo de habilidades sociales como la escucha, la tolerancia, el respeto, que son esenciales para la convivencia social.

Algunos autores (Syahril, 2018) consideran el clima escolar como una situación en la que intervienen todos los actores del centro educativo como el director y los maestros, así como las relaciones entre profesores y de estos con el personal administrativo, estudiantes y padres de familia, llegando a plantearse incluso que el clima influye en un buen proceso de enseñanza-aprendizaje.

Debido al gran impacto que tiene esta variable para lograr una inclusión positiva en la organización educativa, cabe resaltar que en los últimos años, ha habido un aumento considerable en las investigaciones y publicaciones sobre el tema (Nicholas, 2019), lo cual indica la importancia del mismo y la necesidad de profundizar en la comprensión del fenómeno y ampliar su descripción en Colombia.

Muchos de estos estudios han señalado una relación entre el clima existente en el aula y el rendimiento en pruebas de lenguaje (Laurito, Lacoe, Schwartz, Sharkey, \& Ellen, 2019); así como también, el impacto en el aprendizaje, las relaciones entre los estudiantes y el ambiente emocional general de la escuela (Chateadores \& Joo, 2018).

Es así como, algunos autores (Pineda et al., 2019) han determinado la importancia de ciertos factores (Syahril, 2018) para crear y mantener un clima adecuado en el aula, entre los más mencionados están: la relación, el crecimiento/desarrollo, el mantenimiento del sistema personal y el cambio, y la dimensión del entorno físico. Las diversas propuestas realizadas, se pueden agrupar en varios niveles vinculados unos con otros (Gifre \& Esteban, 2012), como se señala a continuación.

En el macrosistema cabe considerar todas las leyes, reglamentos que orientan la labor educativa y que tienen impacto sobre las relaciones humanas. También es importante considerar el respeto a los derechos humanos.

En el macrosistema cabe considerar todas las leyes, reglamentos que orientan la labor educativa y que tienen impacto sobre las relaciones humanas. También es importante considerar el respeto a los derechos humanos.

En el mesosistema es importante tomar en cuenta los estándares educativos, la responsabilidad y organización del talento humano, el presupuesto, la colaboración de los actores, la infraestructura escolar y las actividades organizadas.

Todo proceso de inclusión debe considerarse como parte de un proceso más amplio que involucra tanto a la institución como al entorno en el que se encuentra si se desea un desarrollo sostenible (Echeita Sarrionandia \& Navarro Mateu, 2014). Estos dos aspectos guardan estrecha relación y deben ir de la mano con los principios y valores básicos de colaboración y cooperación y una cultura organizacional y políticas educativas que favorezcan la sostenibilidad.

En el microsistema se puede considerar a los niños y niñas, docentes y personal administrativo que interactúan en la unidad educativa. En cada uno de ellos, las variables sociodemográficas como la edad, el sexo, la religión, el estrato social, el idioma, las condiciones físicas y psicológicas 
propias impactan sobremanera en el clima del aula. También hay que tomar en cuenta el entorno social y los aspectos de ciudadanía como elementos que coadyuvan a un buen desenvolvimiento en la institución.

De la misma manera, se han planteado algunos elementos que son esenciales para desarrollar un clima adecuado para el aprendizaje de todos los niños, tanto los que tienen necesidades educativas como aquellos que no (Shukla et al., 2019). Estos elementos se han denominado como compromiso, la seguridad y el entorno escolar, incluso existen estudios (Starkey, Aber, \& Crossman, 2019) que afirman que el clima escolar modera la violencia en el contexto social.

Un elemento esencial y que tiene gran impacto en el mantenimiento de un buen clima en el aula. es el docente (Reinoso, 2018), quien debe ejercer un rol de gestor eficiente, con una buena comunicación con sus estudiantes y los padres y madres de familia.

Cabe señalar que un estudio realizado (Briones, 2015) no encontró una relación significativa entre el clima del aula y el rendimiento escolar; sin embargo, parece ser que es un factor asociado, como lo muestra otra investigación (Carranza, 2018), en la que se encontró que los conflictos entre los estudiantes $\mathrm{y}$ entre estos y el docente repercuten en el estado de ánimo, la confianza, la colaboración, la comunicación y la disciplina en el aula.

Tanto ha sido el interés generado por esta variable del clima en el aula, que incluso ya se ha propuesto una escala para valorarla, por parte de Moos y Trickett (Prado Delgado, Ramírez Mahecha, \& Ortíz Clavijo, 2011), que consta de tres dimensiones: Relaciones (implicación, afiliación y ayuda); Autorrealización (tareas, competitividad y cooperación) y Estabilidad (organización, claridad, control, cambio e innovación).

Se va a realizar una breve descripción de cada una de estas dimensiones a continuación, por considerarlas como aspectos cruciales para desarrollar una inclusión en el aula, desde una perspectiva de colaboración.

La dimensión de las Relaciones: abarca todas las posibles interacciones entre los actores del hecho educativo: docentes, estudiantes y administrativos participan activamente en intercambios comunicacionales que desarrollan cierto tipo de clima en el aula. Considera tres aspectos: implicación, afiliación y ayuda.

Implicación: valora el interés de los niños por las actividades propuestas por el docente. Por extensión se da importancia a la participación en las actividades y el grado en el cual se involucran en tareas, tanto escolares como extras.

Afiliación: hace referencia al nivel en el cual se establecen relaciones con otras personas, tanto en el ambiente educativo como fuera de él. Se considera que las relaciones al interior de la escuela son claves para desarrollar ciertos valores como la colaboración, la tolerancia y el respeto; sin embargo, estos valores se manifestarán en el entorno social de los chicos.

Esta afiliación se consolida gracias al apoyo que los estudiantes se pueden dar entre ellos, al apoyo que reciben del profesor y el nivel de confianza desarrollado entre ambos que eleva el autoestima (Musitu \& Martínez, 2009) y consolida el respeto en la relación.

Ayuda: es el interés natural y sincero mostrado por el docente, tanto en el aula como en otros espacios de la institución educativa. El docente es el líder de la clase puesto que organiza, planifica, ejecuta y evalúa las actividades realizadas en ella; sin embargo, su actitud hacia los niños y sus características son esenciales a la hora de generar un clima de confianza en el aula.

Así, un estilo educativo democrático (Torío, Peña, \& Rodríguez, 2008) es mucho más adecuado para la educación que uno autoritario, tanto en padres como en maestros. 
En este sentido, el docente, también debe comunicarse con los padres de familia en el caso de que haya algún problema con el hijo o incluso y mejor aún, si no lo hay, con la finalidad de que haya un intercambio adecuado entre familia y escuela (Roiz, 1989), los dos entornos en los que los niños se forman.

La dimensión de la autorrealización: evalúa el crecimiento personal y la orientación a metas (Pineda et al., 2019) aspectos que se potencian gracias a la actitud del docente hacia los estudiantes: los estímulos que les aporta, la confianza que da y la forma en que plantea las actividades son tantas otras condiciones básicas para llevar a cabo la tarea docente y favorecer esta dimensión (Quintero, 2006). Este factor considera los siguientes aspectos: la orientación a las tareas, la competitividad y la cooperación.

La orientación a las tareas: hace referencia a la importancia que el docente da a la realización de las tareas previstas, entendidas como actividades programadas que los estudiantes deben realizar en un tiempo definido y que pretenden consolidar lo aprendido en el aula de clase (Simón Rueda et al., 2015).

La competitividad: se la considera un factor importante en el clima del aula, porque da cuenta del esfuerzo realizado por el estudiante para lograr una buena calificación. Se trata de un esfuerzo positivo y se la entiende como tal, y no como en el sentido negativo de ganar a toda costa. Implica las habilidades para llevar a cabo las tareas previstas y también la actitud que poseen los niños en el momento de cumplirlas; es decir, hacerlo por responsabilidad y no solo por una nota o por cumplir la tarea de manera obligada.

La cooperación: establece el nivel de participación existente en el aula y la forma en que los niños se integran unos con otros para contribuir a la ejecución de las tareas previstas, compartiendo conocimientos y contribuyendo al trabajo en grupo, de ser el caso o dando apoyo a quien lo solicite de ser necesario.
La dimensión de estabilidad: implica la duración de las acciones y actividades en el tiempo de tal forma que se pueda realizar la actividad sin el temor de que se acaba el tiempo o que habrá una mala nota si no se la entrega. Considera varios aspectos como la organización, la claridad, el control, el cambio y la innovación.

La organización: implica la planificación de las actividades en una secuencia ordenada de tal forma que las personas puedan saber lo que antecede y las consecuencias de lo que van haciendo. También debe considerar los recursos disponibles para llevar a cabo la tarea y las personas implicadas en ella. Así, se puede tomar en cuenta a los estudiantes, a los docentes y otros aspectos que hay que establecer como una comunicación bidireccional (Watzlawick, Beavin, (Watzlawick, Beavin, \& Jackson, 1995), el fijarse metas alcanzables, el liderazgo y las normas.

La claridad: implica la existencia de un grado de transparencia aceptable en las actividades que se realizan en el contexto educativo. Esta claridad debe percibirse respecto a aspectos como las reglas y sus consecuencias, las evaluaciones que se van a llevar a cabo, los objetivos que se pretende alcanzar, los temas que se abordarán y la metodología que se pretende usar.

También implica transparentar las acciones de tal forma que los implicados sepan a qué atenerse en la mayor parte de las actividades que se lleven a cabo.

El control: se refiere al nivel en el que el docente revisa $y$ es vigilante atento del cumplimiento adecuado de las normas y reglas establecidas en clase y, también es el garante de la organización que se haya escogido en el aula, puesto que de él depende la gestión de los contenidos, los recursos y el tiempo que se debe dedicar a cada actividad.

La dimensión de los cambios es uno de los aspectos más interesantes del contexto educativo ya que el ser humano siempre está en continuo 
proceso de adaptación a las circunstancias que debe enfrentar. No existe un solo momento en el que la persona se mantenga inalterable ya que siempre existe un hecho o circunstancia que lo va a obligar a adaptarse y, por lo tanto, a cambiar.

La innovación es un proceso por el cual, una persona propone una modificación o adaptación en una parte del currículo o en la metodología de enseñanza. La innovación es como abrir una puerta de las que están disponibles que conducen a una determinada habitación en la que se encontrará con ciertas cosas y no otras (Johnson, 2011), que luego lo conducirán a otros hallazgos y potenciarán ciertos recursos mientras otros pueden irse perdiendo.

De igual manera, también se ha planteado (Arnaiz \& Guirao, 2015) otras formas de evaluar el clima. Así, por ejemplo, en España se ha elaborado el instrumento denomi-nado ACADI (Autoevaluación de Centros para la Atención a la Diversidad desde la Inclusión) con la finalidad de mejorar la calidad y eficacia de la atención en las instituciones educativas, para construir centros cada vez más inclusivos y que acepten la diversidad como parte de su naturaleza misma.

proceso de adaptación a las circunstancias que debe enfrentar. No existe un solo momento en el que la persona se mantenga inalterable ya que siempre existe un hecho o circunstancia que lo va a obligar a adaptarse $\mathrm{y}$, por lo tanto, a cambiar.

La innovación es un proceso por el cual, una persona propone una modificación o adaptación en una parte del currículo o en la metodología de enseñanza. La innovación es como abrir una puerta de las que están disponibles que conducen a una determinada habitación en la que se encontrará con ciertas cosas y no otras (Johnson, 2011), que luego lo conducirán a otros hallazgos y potenciarán ciertos recursos mientras otros pueden irse perdiendo.

De igual manera, también se ha planteado (Arnaiz \& Guirao, 2015) otras formas de evaluar el clima. Así, por ejemplo, en España se ha elaborado el instrumento denomi-nado ACADI (Autoevaluación de Centros para la Atención a la Diversidad desde la Inclusión) con la finalidad de mejorar la calidad y eficacia de la atención en las instituciones educativas, para construir centros cada vez más inclusivos y que acepten la diversidad como parte de su naturaleza misma.

En este proceso, se ha podido identificar algunas debilidades que impiden el desarrollo de una verdadera actividad inclusiva (Arnaiz, Escarbajal, \& Caballero, 2017), señalando fundamentalmente a la política institucional como aspecto esencial y necesario de la inclusión, que de no estar presente, impide la concreción de esta característica en la institución.

En todo este proceso, se debe considerar a un actor clave al que no se puede dejar de lado, debido al impacto que puede tener sobre la concreción de la inclusión en el aula, como es el docente.

\section{El papel del profesor}

Además de sus habilidades y destrezas como docente, el profesor debe tener (Crisol Moya et al., 2015) una excelente capacidad de reflexión para percibir la inclusión y plantear las formas más apropiadas para alcanzarla en el aula y debe participar e involucrarse activamente en un trabajo cooperativo con los demás actores del centro educativo.

Se trata de un verdadero compromiso con la inclusión, que solo se logra cuando el docente ha comprendido sus alcances y limitaciones $\mathrm{y}$ ha desarrollado las habilidades para plantear actividades en su aula y desarrollar en ella, un verdadero clima inclusivo (Crisol Moya et al., 2015).

La actitud es clave en este proceso, puesto que favorecerá o no, el apoyo y colaboración entre los estudiantes, encontrándose así, que si el docente tiene una actitud positiva (Crisol Moya et al., 
2015), también genera un buen nivel de satisfacción y existe un menor grado de exclusión entre los estudiantes. Sin embargo, lo contrario también es cierto, si la actitud del docente es negativa, esto impactará ciertamente sobre el resultado obtenido en el aula y no se favorecerá un clima escolar adecuado para la inclusión.

Para lograr esto, es necesario contar con profesionales capacitados en el tema de la inclusión, con información adecuada acerca de las dimensiones tanto teóricas como prácticas de este proceso y la formación continua en esta área, así como el hecho de contar con pares que lo acompañen en el proceso, puede facilitar la asimilación de los aspectos implicados en la inclusión y contribuir a su desarrollo en el aula y en la institución educativa.

De igual manera, el docente requiere una formación pedagógica y didáctica específica y concreta respecto a temas de inclusión y vinculados a su área de desempeño de tal manera que pueda adaptar la enseñanza a las características de sus estudiantes.

Otro aspecto necesario es que el docente conozca e investigue bien las características de la población con la que va a trabajar (Balongo \& Mérdida, 2015), lo cual permite la construcción de algunos aspectos clave para la inclusión como son: "1) la ayuda entre iguales; 2) el incremento de la motivación; 3) la fuerte implicación emocional que se genera; 4) la atención personalizada que se establece; y 5) la relación entre los discentes y el docente" (p. 146). En este punto, la actitud del docente es clave a la hora de mostrar tolerancia y respeto hacia cada rasgo de los estudiantes. Si él muestra respeto y no admite la segregación, entonces los estudiantes también pueden considerar la inclusión de otras características en el aula.

También se requiere su involucramiento en prácticas investigativas (Parrilla \& Sierra, 2015), que favorezcan el conocimiento y descripción de las características que deben tener para potenciar la inclusión a todo nivel y de ciertas prácticas inclusivas (Álamos et al., 2017) promovidas en otros contextos y que incluyen el uso de cuentos y programas de intervención (López Cobo \& González López, 2012) formulados con el objetivo de mejorar la inclusión en las instituciones educativas.

Para finalizar este punto, el gobierno institucional debe expresar claramente su punto de vista sobre la inclusión y proveer las condiciones que permiten un uso flexible de los recursos por parte de los docentes. Así, ellos sentirán apoyo para su ejercicio profesional y prestarán más fácil su contingente para colaborar con el proceso.

En este sentido, un aspecto institucional que impacta positivamente en el desarrollo de actividades inclusivas y en la introducción de estos aspectos en la institución, es la percepción de los docentes sobre la inclusión y más allá de esto, la asunción sincera del concepto de diversidad en la institución educativa como parte del quehacer docente. Sin embargo, esto todavía es un proceso en construcción como lo demuestra un estudio reciente (Mellado, Chaucono, Hueche, \& Aravena, 2017), en el que se encontró que todavía existen percepciones de prácticas excluyentes que demuestran que aún hay mucho trabajo por realizar, especialmente en relación a la necesidad de crear un currículo inclusivo que promueva los aspectos pedagógicos y didácticos desde esta visión.

Tomando en consideración los múltiples aspectos implicados en el clima del aula, es posible resumirlos (Crisol Moya et al., 2015) en un valor importante: la cooperación combinado con una habilidad esencial como es la colaboración para la resolución de conflictos inevitables en entornos en los que existen marcadas diferencias y la organización de grupos heterogéneos que permiten el contacto más cercano entre niños con diversas características.

También es importante que la institución educativa desarrolle una buena relación con 
padres y madres de familia, que se involucren en las actividades de los hijos y que promuevan también los valores de la inclusión, luego de haberla entendido y asimilado.

\section{La familia}

También debe ser asumida como un agente de inclusión (Crisol Moya et al., 2015). Se la considera como uno de los pilares de la educación general y de la inclusiva con más razón (Bell, Illán, \& Benito, 2010) puesto que allí, se valoran las características de cada uno de los miembros y se establecen las bases sobre las cuales se construyen las primeras experiencias de confianza y de respeto hacia el otro.

El papel de la familia, por lo tanto, es clave para el desarrollo y mantenimiento de un aula inclusiva puesto que su postura afectará profundamente, las actitudes de los niños hacia las personas con otras características diversas. También se requiere la construcción de relaciones positivas con los padres y madres de familia, de tal forma que se involucren en el proceso formativo de sus hijos y sean aliados positivos en la educación y formación de los niños y niñas.

Las familias se desenvuelven en comunidades diversas en las que conviven grupos humanos con características muy diferentes que también deben ser considerados para un trabajo inclusivo.

\section{La comunidad}

Junto a la familia constituye el otro pilar por excelencia de las prácticas inclusivas. Para lograrlo se requiere que también haga cambios estructurales en su entorno para facilitar el acceso de todas las personas con sus características diversas (Crisol Moya et al., 2015) (Bell et al., 2010).

Cuando se alcanza esto, es posible favorecer el aprendizaje cooperativo como un aspecto esencial que permita, a largo plazo, la realización de una verdadera educación inclusiva en un marco ético y de responsabilidad social, que se interesa por el desarrollo integral de todas las personas, cualesquiera que sean sus características.

Para alcanzar estos objetivos, existen experiencias interesantes propuestas en el país, como aquella que destaca la construcción de una "comunidad de aprendizaje" (Beltrán Villamizar, Martínez Fuentes, \& Torrado Duarte, 2015) entendida como "proyectos de transformación social y cultural de un centro educativo y de su entorno, basados en el aprendizaje dialógico, con la finalidad de vincular a toda la comunidad al proceso educativo en espacios concretos, incluyendo el aula de clase" (p. 57). Aspecto que también ha sido destacado en otros estudios (Ortiz, 2018), en los que se verifica el impacto que tiene la posibilidad de inclusión sobre el aprendizaje.

\section{Conclusiones}

Se ha considerado el tema de las aulas inclusivas como entornos que favorecen el acceso de todas las personas a la educación, no solo como cumplimiento de sus derechos esenciales, sino y más importante aún, como consideración de sus características particulares y respeto a sus condiciones especiales.

Se han considerado, además, algunas características del aula inclusiva centradas en la construcción de una actitud de respeto y colaboración entre todas las personas presentes en el aula.

También se ha caracterizado la comunidad y la institución a través de la adopción de una política inclusiva que favorezca las condiciones, tanto económicas como sociales y de percepción que contribuyan a la adopción de una verdadera política de inclusión.

Finalmente, se ha planteado la importancia de la participación de los docentes en el proceso. Son los llamados a mantener un clima escolar inclusivo en el aula y también favorecer las condiciones necesarias para incluir la diversidad 
y asumirla como parte de la existencia misma.

\section{Referencias}

Álamos, P., Cifuentes, O., Milicic, N., Pizarro, M., Rosas, R., Ulloa, D., \& Véliz, S. (2017). Construcción de cuentos: ¿Qué pueden aportar al desarrollo socioemocional inclusivo? Estudios Pedagogicos, 43(1), 7-17. Recuperado de: https://doi.org/10.4067/S07 18-07052017000100001

Arnaiz, P., Escarbajal, A., \& Caballero, C. (2017). El impacto del contexto excolar en la inclusión educativa. Revista de Educación Inclusiva, 10(2), 195-210. Retrieved from http://www.revistaeducacioninclusiva.es/ind ex.php/REI/article/view/334/308

Arnaiz, P., \& Guirao, J. M. (2015). La autoevaluación de centros en España para la atención a la diversidad desde una perspectiva inclusiva: ACADI. Revista Electrónica Interuniversitaria de Formación Del Profesorado, 18(1), 45. Recuperado de: https://doi.org/10.6018/reifop.18.1.214341

Balongo, E., \& Mérdida, R. (2015). El clima de aula en los proyectos de trabajo Crear ambientes de aprendizaje para incluir la diversidad infantil. Perfiles Educativos, XXXVIII(152), 146-162.

Bell, R., Illán, N., \& Benito, J. (2010). Familia Escuela - Comunidad: pilares para la inclusión. Revista Interuniversitaria de Formación Del Profesorado, 24(3), 47-57.

Beltrán Villamizar, Y. I., Martínez Fuentes, Y. L., \& Torrado Duarte, O. E. (2015). Creación de una comunidad de aprendizaje: una experiencia de educación inclusiva en Colombia. Revista Encuentros, 13(2). Recuperado de: https://doi.org/10.15665/re.v13i2.498

Briones, G. (2015). El clima escolar y su incidencia en el rendimiento académico de los estudiantes de Educación General Básica Superior del Colegio Fiscal Provincia de Bolívar de la ciudad de Guayaquil, período 2014-2015. Universidad de Guayaquil.

Carranza, M. L. (2018). El clima escolar en una escuela oficial rural mixta de Río Hondo, Zacapa. Universidad Rafael Landivar. Universidad Rafael Landivar.

Chateadores, S., \& Joo, H. (2018). Dimensiones del clima escolar asociado con reportes de intimidación y victimización en un entorno de atención a jóvenes predominantemente latina. International Journal of Psycho Educational Sciences, 7(1), 52-67.

Crisol Moya, E., Martínez, J., \& El Homrani, M. (2015). Hacia un aula inclusiva. Condiciones didácticas y organizativas. Dialógica: Revista Multidisciplinaria, 12(2), 217-258.

Echeita Sarrionandia, G., \& Navarro Mateu, D. (2014). Educación inclusiva y desarrollo sostenible: una llamada urgente a pensarlas juntas. Edetania: Estudios y Propuestas Socio-Educativas, (46), 141-162.

Gifre, M., \& Esteban, M. (2012). Consideraciones educativas de la perspectiva ecológica de Urie Bronfenbrenner. Contextos Educativos, 15, 79-92. Recuperado de: https://doi.org/10.18172/con.656

González Ledesma, V. G. (2012). Teorías del desarrollo humano, 1-27. Retrieved from https://portalacademico.cch.unam.mx/reposi torio-de-sitios/experimentales/psicologia $2 / p s$ cII/MD1/MD1-L/teorias_desarrollo.pdf

Guerrero, H., Crissien, T., \& Aniagua, R. (2017). Proyectos Educativos Institucionales Colombianos (PEI): Educación Inclusiva a través de la autoevaluación. Institución Educativa La Vecina. Opción, Año, 34, 218-266. Retrieved from https://www.redalyc.org/pdf/310/310549910 09.pdf 
Johnson, S. (2011). Las buenas ideas. Una historia natural de la innovación. (T. Publicaciones, Ed.). Madrid.

Laurito, A., Lacoe, J., Schwartz, A. E., Sharkey, P., \& Ellen, I. (2019). El clima escolar y el impacto del crimen en las calificaciones de Barrio. Revista de Ciencias Sociales, 5(2), 141-166.

Lázaro-Visa, S., Palomera, R., Briones, E., Fernández-Fuentes, A., \& Fernández-Rouco, N. (2019). Satisfacción: Las competencias personales y el ambiente escolar como factores protectores. Frontiers in Psychology, 10, $1-11$.

López Cobo, I., \& González López, I. (2012). Generación de entornos inclusivos desde la mejora de la eficacia escolar. Revista de Educación Inclusiva, 5(2), 9-23.

Mellado, M. E., Chaucono, J. C., Hueche, M., \& Aravena, O. (2017). Percepciones sobre la educación inclusiva del profesorado de una escuela con Programa de Integración. Revista Educación, 41(1), 1-14. Recuperado de: https://doi.org/10.15517/revedu.v41i1.21597

Montáncez, M. L., Jornet, J., Perales, M., Carrillo, S., \& Wilches, S. (2017). Educación Inclusiva. Maracaibo: Universidad del Zulia.

Muñoz, M. T., Lucero, B., Cornejo, C., Muñoz, P., \& Araya, N. (2014). Convivencia y clima escolar en una comunidad educativa inclusiva de la Provincia de Talca, Chile. Revista Electronica de Investigacion Educativa, 16 (2), 16-32.

Musitu, G., \& Martínez, B. (2009). Familia y escuela. Una complicidad necesaria en la prevención de las drogodependencias. In Congreso "Hablemos de Drogas" (pp. 1-12). Barcelona: CosmoCaixa Barcelona.

Nicholas, N. (2019). Ambiente escolar: mapeo y análisis de papers. Ambiente Educativo, 12
(1), 50-67.

Ortiz Granja, D. (2015). The intercultural education: the challenge of the unity in diversity. Sophia, 18(1), 91-110. https://doi.org/10.17163/soph.n18.2015.05

Ortiz Granja, D. N., \& Joaqui Robles, D. (2017). Educación como práctica social: la cuestión del otro y su reconocimiento. Sophía, (23), 169. Recuperado de:

https://doi.org/10.17163/soph.n23.2017.07

Ortiz, I. (2018). Escuelas inclusivas en el contexto de segregación social del sistema escolar chileno. Calidad En La Educación, (42), 93. Recuperado de: https://doi.org/10.31619/caledu.n42.53

Parrilla, Á., \& Sierra, S. (2015). Construyendo una investigación inclusiva en torno a las distintas transiciones educativas. Revista Electrónica Interuniversitaria de Formación Del Profesorado, 18(1), 161-175.

Pineda, S., Granizo, C., \& Zúniga, J. (2019). Gestión Pedagógica: Clima social en el aula, desde la percepción de estudiantes y profesores. Compás (Primera). Guayaquil: Ediciones Grupo Compás.

Prado Delgado, V. M., Ramírez Mahecha, M. L., \& Ortíz Clavijo, M. S. (2011). Adaptación y validación de la escala de clima social escolar (CES). Actualidades Investigativas En Educación, 10(2). Recuperado de: https://doi.org/10.15517/aie.v10i2.10121

Quintero, R. (2006). Una teoría sobre la motivación humana. Psychological Review, 50, 370-396.

Reinoso, H. (2018). Clima escolar en estudiantes pertenecientes al grado sexo del liceo de Cervantes Norte. Retrieved from http://informaticareuven.com.co/reuven/ejer 623.html 
Rodríguez, A. (2018). Expansión postmoderna tecnológica, escuela inclusiva tecnológica. Retos XXI, 2, 5-12.

Roiz, M. (1989). La familia, desde la Teoría de la comunicación de palo alto. Reis, (48), 117. Recuperado de: https://doi.org/10.2307/40183464

Shukla, K. D., Waasdorp, T. E., Lindstrom Johnson, S., Orozco Solis, M. G., Nguyen, A. J., Rodríguez, C., \& Bradshaw, C. P. (2019). Does School Climate Mean the Same Thing in the United States as in Mexico? A Focus on Measurement Invariance. Journal of Psychoeducational Assessment, 37(1), 5-68.Recuperado de: https://doi.org/10.1177 /0734282917731459

Simón Rueda, C., Sandoval Mena, M., Echeita Sarrionandia, G., Calero Gil, C., Nuñez Gutiérrez de San Miguel, B., Pérez García, M. M., \& García de la Torre, A. B. (2015). Transformando la "gramática escolar" para ser más inclusivos: la experiencia de tres centros educativos. Contextos Educativos. Revista de Educación, 0(19), 7.

Recuperado de: https://doi.org/10.18172/con.2784
Starkey, L., Aber, J. L., \& Crossman, A. (2019). Risk or resource: Does school climate moderate the influence of community violence on children's social-emotional development in the Democratic Republic of Congo? Developmental Science, 1-16. Recuperado de: https://doi.org/10.1111/desc. 12845

Syahril, H. (2018). Mejorar el clima de la escuela para una mejor gestión de la calidad educativa. Revista de Ciencias de La Educación y El Aprendizaje, 1(1), 16-22.

Terrón-Caro, T., Cárdenas-Rodríguez, R., \& Rodríguez-Casado, R. (2017). Educación Intercultural Inclusiva. Pedagogía Social, (29), 25-40.

Torío, S., Peña, J., \& Rodríguez, M. del C. (2008). Estilos educativos parentales. Revisión bibliográfica y reformulación teórica. Teoria de La Educacion, 20, 151-178.

Watzlawick, P., Beavin, J., \& Jackson, D. (1995). Teoría de la Comunicación Humana (Décima). Barcelona: Herder. 


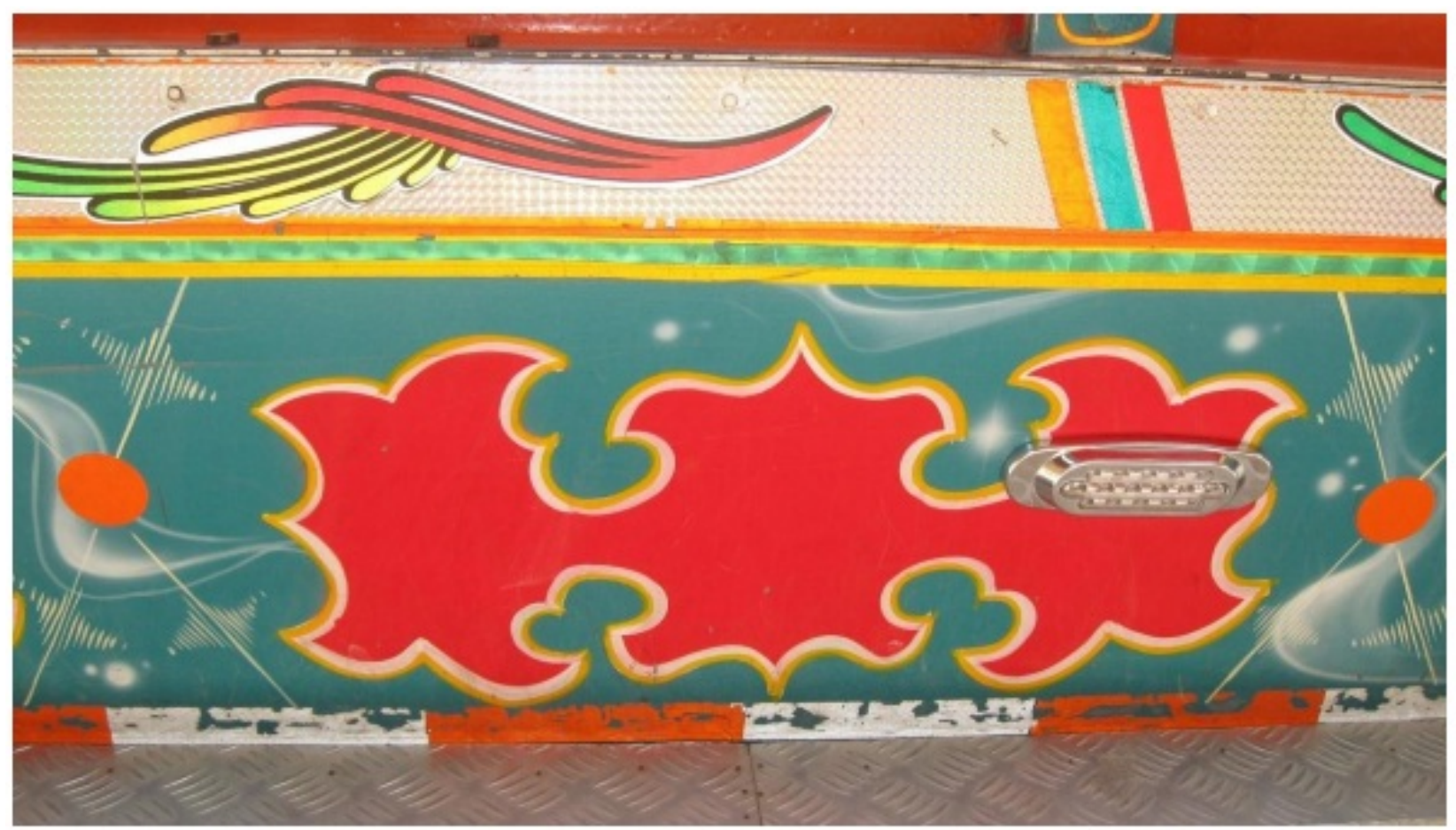

Número de la obra: 79

Título: Chiva "El Lucerito"

Autora: Margarita Chávarro Castro

Lugar: Municipio La Plata, Huila

Fecha de captura: Abril de 2013

Técnica: Fotografía digital

Fuente: Colección Margarita Chávarro Castro 